\title{
Fibre-Reinforced Generalized Thermoelastic Medium Subjected to Gravity Field
}

\author{
Fatimah S. Bayones' ${ }^{1}$, Nahed S. Hussien ${ }^{1,2}$ \\ ${ }^{1}$ Mathematics Department, Faculty of Science, Taif University, Taif, Saudi Arabia \\ ${ }^{2}$ Mathematics Department, Faculty of Science, Cairo University, Cairo, Egypt \\ Email: f.s.bayones@hotmail.com, hussein.nahed@yahoo.com
}

Received 24 February 2015; accepted 20 July 2015; published 23 July 2015

Copyright (C) 2015 by authors and Scientific Research Publishing Inc.

This work is licensed under the Creative Commons Attribution International License (CC BY). http://creativecommons.org/licenses/by/4.0/

(c) $($ i) 0 pen Access

\begin{abstract}
The present paper is aimed at studying the effects of thermal relaxation time and gravity of a twodimensional problem with anisotropic semi-infinite solid media with the deformation of fiberreinforced generalized thermoplastic. The harmonic function techniques are used. The thermal stresses, temperature and displacement have been obtained. These expressions are calculated numerically for two values of elastic constants for mediums $M_{1}$ and $M_{2}$ respectively. The results obtained are displayed by graphs to clear the phenomena physical meaning. The results indicate that the effect of gravity, relaxation times and parameters of fibre-reinforced of the material medium are very pronounced.
\end{abstract}

\section{Keywords}

Fibre-Reinforced Medium, Thermal Relaxations, Viscosity, Thermoelastic, Gravity

\section{Introduction}

The classical theory of thermoelasticity has been generalized and modified into various thermoelastic models that run under the label of "hyperbolic thermoelasticity". The linear theory of elasticity of paramount importance in the stress analysis of steel is the commonest engineering structural material. To a lesser extent, linear elasticity describes the mechanical behavior of the other common solid materials, e.g. concrete, wood and coal. Thermoelasticity theories, which admit a finite speed for thermal signals, have been receiving a lot of attention for the past four decades. In contrast to the conventional coupled thermoelasticity theory based on a parabolic heat equation, Biot [1], which predicts an infinite speed for the propagation of heat, these theories involve a hyperbolic heat equation and are referred to as generalized thermoelasticity theories.

The notation hyperbolic reflects the fact that thermal waves are modelled, avoiding the physical paradox of 
the infinite propagation speed of the classical model. At present, there are several theories of the hyperbolic thermoelasticity. The first was developed by Lord and Shulman [2] who obtained a wave-type heat equation by postulating a new law of heat conduction to replace the classical Fourier's law. This new law contains the heat flux vector as well as its time derivative. It also contains a new constant that acts as a relaxation time. The second was developed by Green and Lindsay [3]. Tanaka, et al. [4] studied the application of boundary element method to 3-D problems of coupled thermoelasticity. A generalization of this inequality was proposed by Green and Laws [5]. Abd-Alla, et al. [6] discussed the transient thermal stresses in atransversely isotropic infinite circular cylinder. Effect of initial stress on generalized thermoelastic problem in an infinite circular cylinder is discussed by El-Naggar and Abd-Alla [7]. Effect of the rotation on an infinite generalized magneto-thermoelastic diffusion body with a spherical cavity was discussed by Abd-Alla, et al. [8]. Ezzat and Youssef [9] studied the generalized magneto-thermoelasticity in a perfectly conducting medium. Abd-Alla and Bayones [10] studied effect of rotation and initial stress on generalized-thermoelastic problem in an infinite circular cylinder. Dai and Wang [11] studied the magneto-elastodynamic stress and perturbation of magnetic field vector in an orthotropic laminated hollow cylinder. Abd-Alla, et al. [12] studied thermal stresses in a non-homogeneous orthotropic elastic multilayered cylinder.

Fibre-reinforced composites are used in a variety of structures due to their low weight and high strength. A continuum model is used to explain the mechanical properties of such materials. Craig and Hart [13] studied the stress boundary value problem for finite plane deformation of a fibre-reinforced material. Sengupta and Nath [14] discussed the problem of surface waves in a fibre-reinforced anisotropic elastic media. Singh and Singh

[15] discussed the reflection of plane waves at the free surface of a fibre-reinforced elastic half-space. Singh [16] discussed the wave propagation in an incompressible transversely isotropic fibre-reinforced elastic media. Singh [17] studied the effects of anisotropy on reflection coefficients of plane waves in fibre-reinforced thermoelastic solid. Kumar and Gupta [18] investigated a source problem in fibre-reinforced anisotropic generalized thermoelastic solid under acoustic fluid layer. Alla et al. [19] investigated propagation of Rayleigh waves in generalized magneto-thermoplastic orthotropic material under initial stress and gravity field. Thermal stresses in an infinite circular cylinder have been investigated by Abd-Alla et al. [20]. Abd-Alla and Mahmoud [21] studied the magneto-thermoelastic problem in rotatin non-homogeneous orthotropic hollow cylinder under the hyperbolic heat conduction model. Abd-Alla and Ahmed [22] investigated the Rayleigh waves in an orthotropic thermoelastic medium under gravity field and initial stress. Rayleigh waves in a magnetoelastic half-space of orthotropic material under the influence of initial stress and gravity field were investigated by Abd-Alla et al. [23]. Elnaggar and Abd-Alla [24] studied Rayleigh waves in magneto-thermo-microelastic half-space under initial stress. AbdAlla and Ahmed [25] discussed Rayleigh waves in an orthotropic thermoelastic medium under gravity field and initial stress. Effect of rotation and initial stress on an infinite generalized magneto-thermoelastic diffusion body with a spherical cavity is investigated by Abd-Alla and Abo-Dahab [26].

In this paper then we study the effect of thermal relaxation time and gravity on anisotropic semi-infinite solids with the deformation of fibre-reinforced generalized thermoelastic. The thermal stresses, temperature and displacement have been obtained. Furthermore, the displacement, temperature and stresses are computed numerically in each of these cases and illustrated graphically. The results indicate that the effect of thermal relaxation time and gravity on the thermal stresses, temperature and displacement are very pronounced.

\section{Formulation of the Problem}

Consider a homogeneous thermally conducting transversely fibre-reinforced medium with thermal relaxation time and gravity. Let us consider a system of anisotropic Cartesian axes Oxyz, $M_{1}$ and $M_{2}$ be two firesreinforced elastic anisotropic semi-infinite solid media. Let $\mathrm{O}$ be the any point on the plane boundary and $\mathrm{Oz}$ points vertically downward to the medium $M_{1}$. Further the mechanical properties of $M_{1}$ are different from those of $M_{2}$. These media extend to an infinite great distance from the origin and are separated by a plane horizontal boundary and $M_{2}$ is to be taken above $M_{1}$. Further let us assume that $u, v, w$ are the components of displacements at any point $(x, 0, z)$ at any time $t$. It is also assumed that gravitational field produces a hydrostatic initial stress is produced by a slow process of creep where the shearing stresses tend to become smaller or vanish after a long period of time.

The equilibrium conditions of the initial stress field [20] 


$$
\frac{\partial \tau}{\partial x}=0, \quad \frac{\partial \tau}{\partial z}+\rho g=0
$$

The stress-temperature equation is given by [21]:

$$
K T_{i j}=\rho c_{v} \frac{\partial}{\partial t}\left(1+\tau_{2} \frac{\partial}{\partial t}\right) T+\gamma T_{0} \frac{\partial}{\partial t}\left(1+\tau_{2} \delta \frac{\partial}{\partial t}\right) \underline{\nabla} \cdot \boldsymbol{u}, i, j=1,2,3 .
$$

The dynamical equations of motion for three-dimensional elastic solid medium under the influence of initial stress and gravity [22]

$$
\begin{aligned}
& \frac{\partial \tau_{11}}{\partial x}+\frac{\partial \tau_{12}}{\partial y}+\frac{\partial \tau_{13}}{\partial z}+\rho g \frac{\partial w}{\partial x}=\rho \frac{\partial^{2} u}{\partial t^{2}}, \\
& \frac{\partial \tau_{12}}{\partial x}+\frac{\partial \tau_{22}}{\partial y}+\frac{\partial \tau_{23}}{\partial z}+\rho g \frac{\partial w}{\partial y}=\rho \frac{\partial^{2} v}{\partial t^{2}}, \\
& \frac{\partial \tau_{13}}{\partial x}+\frac{\partial \tau_{23}}{\partial y}+\frac{\partial \tau_{33}}{\partial z}-\rho g\left(\frac{\partial u}{\partial x}+\frac{\partial v}{\partial y}\right)=\rho \frac{\partial^{2} w}{\partial t^{2}},
\end{aligned}
$$

The constitutive equations for a fibre-reinforced linearly thermoelastic anisotropic medium with respect to a preferred direction $\boldsymbol{a}$ are [23]

$$
\begin{aligned}
\tau_{i j}= & \lambda e_{k k} \delta_{i j}+2 \mu_{T} e_{i j}+\alpha\left(a_{k} a_{m} e_{k m} \delta_{i j}+e_{k k} a_{i} a_{j}\right) \\
& +2\left(\mu_{L}-\mu_{T}\right)\left(a_{i} a_{k} e_{k j}+a_{j} a_{k} e_{k i}\right)+\beta\left(a_{k} a_{m} e_{k m} a_{i} a_{j}\right)-\gamma\left(T+\tau_{1} \dot{T}\right) \delta_{i j}
\end{aligned}
$$

where $\tau$ is a function of depth, $\rho$ is density of the material, $K$ is thermal conductivity, $c_{v}$ is specific heat of the material per unit mass, $\tau_{1}, \tau_{2}$ are the thermal relaxation parameters, $\gamma=\alpha_{t}\left(3 \lambda+2 \mu_{T}\right), \alpha_{t}$ is the coefficient of linear thermal expansion, $\lambda, \mu_{T}$ are elastic parameters, $\theta$ is the absolute temperature, $T_{0}$ is the reference temperature solid, $T$ is the temperature difference $\left(\theta-T_{0}\right), g$ be the acceleration due to gravity and $\tau_{i j}=\tau_{j i}, \forall i, j$ are the stress components, $e_{i j}=\frac{1}{2}\left(u_{i, j}+u_{j, i}\right)$ are components of strain; $\alpha, \beta$, $\left(\mu_{L}-\mu_{T}\right)$ are reinforced anisotropic elastic parameters; $\boldsymbol{a}=\left(a_{1}, a_{2}, a_{3}\right), a_{1}^{2}+a_{2}^{2}+a_{3}^{2}=1$. If $\boldsymbol{a}$ has components that are (1, $0,0)$ so that the preferred direction is the $\mathrm{x}$ - axis, (7) can be written as follows:

$$
\begin{aligned}
& \tau_{11}=\left(\lambda+2 \alpha+4 \mu_{L}-2 \mu_{T}+\beta\right) e_{11}+(\lambda+\alpha) e_{22}+(\lambda+\alpha) e_{33}-\gamma\left(T+\tau_{1} \dot{T}\right), \\
& \tau_{22}=(\lambda+\alpha) e_{11}+\left(\lambda+2 \mu_{T}\right) e_{22}+\lambda e_{33}-\gamma\left(T-\tau_{1} \dot{T}\right), \\
& \tau_{33}=(\lambda+\alpha) e_{11}+\lambda e_{22}+\left(\lambda+2 \mu_{T}\right) e_{33}-\gamma\left(T-\tau_{1} \dot{T}\right), \\
& \tau_{23}=2 \mu_{T} e_{23}, \tau_{13}=2 \mu_{L} e_{13}, \tau_{12}=2 \mu_{L} e_{12} .
\end{aligned}
$$

Introducing Equations (7) into Equations (3)-(5), we have

$$
\begin{gathered}
\left(\lambda+2 \alpha+4 \mu_{L}-2 \mu_{T}+\beta\right) \frac{\partial^{2} u}{\partial x^{2}}+\left(\lambda+\alpha+\mu_{L}\right) \frac{\partial^{2} w}{\partial x \partial z}+\mu_{L} \frac{\partial^{2} u}{\partial z^{2}}+\rho g \frac{\partial w}{\partial x}-\gamma\left(1+\tau_{1} \frac{\partial}{\partial t}\right) \frac{\partial T}{\partial x}=\rho \frac{\partial^{2} u}{\partial t^{2}}, \\
\left(\mu_{L}-\mu_{T}\right) \frac{\partial^{2} v}{\partial x^{2}}+\mu_{T}\left(\frac{\partial^{2}}{\partial x^{2}}+\frac{\partial^{2}}{\partial z^{2}}\right) v=\rho \frac{\partial^{2} v}{\partial t^{2}} \\
\mu_{L} \frac{\partial^{2} w}{\partial x^{2}}+\left(\lambda+\alpha+\mu_{L}\right) \frac{\partial^{2} u}{\partial x \partial z}+\left(\lambda+2 \mu_{T}\right) \frac{\partial^{2} w}{\partial z^{2}}-\rho g \frac{\partial u}{\partial x}-\gamma\left(1+\tau_{1} \frac{\partial}{\partial t}\right) \frac{\partial T}{\partial z}=\rho \frac{\partial^{2} w}{\partial t^{2}}
\end{gathered}
$$

By Helmholtz's theorem [24], the displacement vector $\boldsymbol{u}$ can be written in the displacement potentials $\phi$ and $\psi$ form, as

$$
\boldsymbol{u}=\operatorname{grad} \phi+\operatorname{curl} \psi, \boldsymbol{\psi}=(0, \psi, 0)
$$

which reduces to 


$$
u=\frac{\partial \phi}{\partial x}-\frac{\partial \psi}{\partial z}, w=\frac{\partial \phi}{\partial z}+\frac{\partial \psi}{\partial x} .
$$

Now using (12) in (8) and (9) we obtain the following wave equation in $M_{1}$ satisfied by $\phi$ and $\psi$ as:

$$
\begin{gathered}
\left(\lambda+2 \alpha+4 \mu_{L}-2 \mu_{T}+\beta\right) \frac{\partial^{2} \phi}{\partial x^{2}}+\left(\lambda+\alpha+2 \mu_{L}\right) \frac{\partial^{2} \phi}{\partial z^{2}}+\rho g \frac{\partial \psi}{\partial x}-\gamma\left(1+\tau_{1} \frac{\partial}{\partial t}\right) T=\rho \frac{\partial^{2} \phi}{\partial t^{2}} \\
\left(\mu_{L}-\mu_{T}\right) \frac{\partial^{2} v}{\partial x^{2}}+\mu_{T}\left(\frac{\partial^{2}}{\partial x^{2}}+\frac{\partial^{2}}{\partial z^{2}}\right) v=\rho \frac{\partial^{2} v}{\partial t^{2}} \\
\left(\alpha+3 \mu_{L}-2 \mu_{T}+\beta\right) \frac{\partial^{2} \psi}{\partial x^{2}}+\mu_{L} \frac{\partial^{2} \psi}{\partial z^{2}}-\rho g \frac{\partial \phi}{\partial x}=\rho \frac{\partial^{2} \psi}{\partial t^{2}}
\end{gathered}
$$

Substituting from Equation (12) into Equation (2), we obtain:

$$
K \nabla^{2} T=\rho c_{v} \frac{\partial}{\partial t}\left(1+\tau_{2} \frac{\partial}{\partial t}\right) T+\gamma T_{0} \frac{\partial}{\partial t}\left(1+\tau_{2} \delta \frac{\partial}{\partial t}\right) \nabla^{2} \phi
$$

where, $\nabla^{2}=\frac{\partial^{2}}{\partial x^{2}}+\frac{\partial^{2}}{\partial z^{2}}$, and similar relations in $M_{2}$ with $\rho, \lambda, \alpha, \mu_{L}, \beta$ replaced by $\rho^{\prime}, \lambda^{\prime}, \alpha^{\prime}, \mu_{L}^{\prime}, \beta^{\prime}$.

\section{Solution of the Problem}

Assuming a simple harmonic time dependent factor for all the quantities and omitting the factor $\mathrm{e}^{\mathrm{i} \omega(x-c t)}$ as follows:

$$
(\phi, \psi, v, T)=[\Phi(z), \Psi(z), V(z), \Theta(z)] \mathrm{e}^{i \omega(x-c t)} .
$$

Using Equations (17) in (13)-(16), we get a set of differential equations for medium $M_{1}$ as follows:

$$
\begin{aligned}
& \left(D^{2}+m_{1}^{2}\right) \Phi+m_{2}^{2} \Psi-m_{3}^{2} \Theta=0 \\
& \left(D^{2}+m_{4}^{2}\right) \Psi-m_{5}^{2} \Phi=0 \\
& \left(D^{2}+m_{6}^{2}\right) V=0 \\
& \left(D^{2}+A_{1}^{2}\right) \Theta+A_{2}^{2} \Phi=0 .
\end{aligned}
$$

where

$$
\begin{aligned}
& \Gamma_{1}=\frac{\lambda+2 \alpha+4 \mu_{L}-2 \mu_{T}+\beta}{\rho}, \Gamma_{2}=\frac{\alpha+\lambda+2 \mu_{L}}{\rho}, \Gamma_{3}=\frac{\alpha+3 \mu_{L}-2 \mu_{T}+\beta}{\rho} \\
& \Gamma_{4}=\frac{\mu_{L}}{\rho}, \Gamma_{5}=\frac{\mu_{T}}{\rho}, m_{1}^{2}=\frac{\omega^{2}\left(c^{2}-\Gamma_{1}\right)}{\Gamma_{2}}, m_{2}^{2}=\frac{i \omega g}{\Gamma_{2}}, m_{3}^{2}=\frac{L}{\Gamma_{2}}, m_{4}^{2}=\frac{\omega^{2}\left(c^{2}-\Gamma_{3}\right)}{\Gamma_{4}}, \\
& m_{5}^{2}=\frac{i \omega g}{\Gamma_{4}}, m_{6}^{2}=\frac{\omega^{2}\left(c^{2}-\Gamma_{4}\right)}{\Gamma_{5}}, L=\frac{\gamma\left(1-i \omega c \tau_{1}\right)}{\rho}, A_{1}^{2}=\frac{\omega^{2}-i \omega c \rho c_{v}\left(1-i \omega c \tau_{2}\right)}{K}, \\
& A_{2}^{2}=\frac{i \omega c \gamma T_{0}\left(1-i \omega c \delta \tau_{2}\right)}{K}, D=\frac{\mathrm{d}}{\mathrm{d} z} .
\end{aligned}
$$

and those for the medium $M_{2}$ are given by

$$
\begin{aligned}
& \left(D^{2}+m_{1}^{\prime 2}\right) \Phi+m_{2}^{\prime 2} \Psi-m_{3}^{\prime 2} \Theta=0 \\
& \left(D^{2}+m_{4}^{\prime 2}\right) \Psi-m_{5}^{\prime 2} \Phi=0 \\
& \left(D^{2}+m_{6}^{\prime 2}\right) V=0
\end{aligned}
$$




$$
\left(D^{2}+A_{1}^{\prime 2}\right) \Theta+A_{2}^{\prime 2} \Phi=0
$$

where

$$
\begin{aligned}
& \Gamma_{1}^{\prime}=\frac{\lambda^{\prime}+2 \alpha^{\prime}+4 \mu_{L}^{\prime}-2 \mu_{T}^{\prime}+\beta^{\prime}}{\rho^{\prime}}, \Gamma_{2}^{\prime}=\frac{\alpha^{\prime}+\lambda^{\prime}+2 \mu_{L}^{\prime}}{\rho^{\prime}}, \Gamma_{3}^{\prime}=\frac{\alpha^{\prime}+3 \mu_{L}^{\prime}-2 \mu_{T}^{\prime}+\beta^{\prime}}{\rho^{\prime}}, \\
& \Gamma_{4}^{\prime}=\frac{\mu_{L}^{\prime}}{\rho^{\prime}}, \Gamma_{5}^{\prime}=\frac{\mu_{T}^{\prime}}{\rho^{\prime}}, m_{1}^{\prime 2}=\frac{\omega^{2}\left(c^{2}-\Gamma_{1}^{\prime}\right)}{\Gamma_{2}^{\prime}}, m_{2}^{\prime 2}=\frac{i \omega g}{\Gamma_{2}^{\prime}}, m_{3}^{\prime 2}=\frac{L^{\prime}}{\Gamma_{2}^{\prime}}, m_{4}^{\prime 2}=\frac{\omega^{2}\left(c^{2}-\Gamma_{4}^{\prime}\right)}{\Gamma_{3}^{\prime}}, \\
& m_{5}^{\prime 2}=\frac{i \omega g}{\Gamma_{3}^{\prime}}, m_{6}^{\prime 2}=\frac{\omega^{2}\left(c^{2}-\Gamma_{4}^{\prime}\right)}{\Gamma_{5}^{\prime}}, L^{\prime}=\frac{\gamma^{\prime}\left(1-i \omega c \tau_{1}\right)}{\rho^{\prime}} A_{1}^{\prime 2}=\frac{\omega^{2}-i \omega c \rho^{\prime} c_{v}\left(1-i \omega c \tau_{2}\right)}{K}, \\
& A_{2}^{\prime 2}=\frac{i \omega c \gamma^{\prime} T_{0}\left(1-i \omega c \delta \tau_{2}\right)}{K} .
\end{aligned}
$$

Eliminating the temperature $\Theta$ from Equations (22) and (25), we obtain

$$
\left[D^{4}+\left(A_{1}^{2}+m_{1}^{2}\right) D^{2}+A_{1}^{2} m_{1}^{2}+m_{3}^{2} A_{2}^{2}\right] \Phi+m_{2}^{2}\left(D^{2}+A_{1}^{2}\right) \Psi=0,
$$

Using Equation (19) and Equation (26), we get

$$
\left[D^{6}+Q_{1} D^{4}+Q_{2} D^{2}+Q_{3}\right] \Phi=0
$$

Equations (22)-(25) tend to the solutions

$$
\begin{aligned}
& \Phi=\sum_{j=1}^{3}\left[B_{j} \mathrm{e}^{i \omega \lambda_{j} z}+C_{j} \mathrm{e}^{-i \omega \lambda_{j} z}\right] \\
& \Psi=\sum_{j=1}^{3}\left[D_{j} \mathrm{e}^{i \omega \lambda_{j} z}+E_{j} \mathrm{e}^{-i \omega \lambda_{j} z}\right] \\
& V=F \mathrm{e}^{i \omega m_{7} z}+G \mathrm{e}^{-i \omega m_{6} z}
\end{aligned}
$$

where the constants $D_{j}$ and $E_{j}$ are related with the constants $B_{j}$ and $C_{j}$ in the form

$$
\begin{aligned}
& D_{j}=N_{j} B_{j}, E_{j}=N_{j} C_{j}, \quad j=1,2,3 \\
& N_{j}=\frac{m_{5}^{2}}{\left[m_{4}^{2}-\omega^{2} \lambda_{j}^{2}\right]} .
\end{aligned}
$$

From Equations (28) and (29) in Equation (18), we get

$$
\Theta=\frac{1}{m_{3}^{2}} \sum_{j=1}^{3}\left(m_{1}^{2}-\omega^{2} \lambda_{j}^{2}+m_{2}^{2} N_{j}\right)\left[B_{j} \mathrm{e}^{\mathrm{i} \omega \lambda_{j} z}+C_{j} \mathrm{e}^{-i \omega \lambda_{j} z}\right]
$$

where, $\lambda_{j}, j=1,2,3$ are taken to be the complex roots of equation

$$
\begin{gathered}
\lambda^{6}+Q_{1} \lambda^{4}+Q_{2} \lambda^{2}+Q_{3}=0, \\
Q_{1}=m_{4}^{2}+m_{1}^{2}+A_{1}^{2}, Q_{2}=A_{1}^{2}\left(m_{4}^{2}+m_{1}^{2}\right)+m_{1}^{2} m_{4}^{2}+m_{3}^{2} A_{2}^{2}+m_{5}^{2} m_{2}^{2}, \\
Q_{3}=A_{1}^{2}\left(m_{4}^{2} m_{1}^{2}+m_{2}^{2} m_{5}^{2}\right)+m_{3}^{2} m_{4}^{2} A_{2}^{2} .
\end{gathered}
$$

Since the displacement at $z \rightarrow \infty$ should be zero, hence for medium $M_{1}$

$$
\begin{aligned}
& \Phi=\sum_{j=1}^{3}\left[C_{j} \mathrm{e}^{-i \omega \lambda_{j} z}\right], \\
& \Psi=\sum_{j=1}^{3}\left[N_{j} C_{j} \mathrm{e}^{-i \omega \lambda_{j} z}\right],
\end{aligned}
$$




$$
\begin{gathered}
V=G \mathrm{e}^{-i \omega m_{6} z} \\
\Theta=\frac{1}{m_{3}^{2}} \sum_{j=1}^{3}\left(m_{1}^{2}-\omega^{2} \lambda_{j}^{2}+m_{2}^{2} N_{j}\right)\left[C_{j} \mathrm{e}^{-i \omega \lambda_{j} z}\right] .
\end{gathered}
$$

Finally from Equation (17), we have

$$
\begin{gathered}
\phi=\sum_{j=1}^{3}\left[C_{j} \mathrm{e}^{i \omega\left(x-c t-\lambda_{j} z\right)}\right], \\
\psi=\sum_{j=1}^{3}\left[N_{j} C_{j} \mathrm{e}^{i \omega\left(x-c t-\lambda_{j} z\right)}\right], \\
v=G \mathrm{e}^{i \omega\left(-m_{6} z+x-c t\right)}, \\
T=\frac{1}{m_{3}^{2}} \sum_{j=1}^{3}\left(m_{1}^{2}-\omega^{2} \lambda_{j}^{2}+m_{2}^{2} N_{j}\right)\left[C_{j} \mathrm{e}^{i \omega\left(x-c t-\lambda_{j} z\right)}\right] .
\end{gathered}
$$

From Equations (39)-(42), we have

$$
\begin{gathered}
u=i \omega \sum_{j=1}^{3}\left[1+N_{j} \lambda_{j}\right] C_{j} \mathrm{e}^{i \omega\left(x-c t-\lambda_{j} z\right)} \\
w=i \omega \sum_{j=1}^{3}\left[N_{j}-\lambda_{j}\right] C_{j} \mathrm{e}^{i \omega\left(x-c t-\lambda_{j} z\right)}
\end{gathered}
$$

And the stress components are

$$
\begin{aligned}
\tau_{11}= & \omega^{2} \sum_{j=1}^{3}\left\{(\lambda+\alpha)\left(\lambda_{j} N_{j}-\lambda_{j}^{2}\right)-\left(\lambda+2 \alpha+4 \mu_{L}-2 \mu_{T}+\beta\right)\left(1+N_{j} \lambda_{j}\right)\right. \\
& \left.-\frac{\gamma\left(1-i \omega c \tau_{1}\right)\left(m_{1}^{2}-\omega^{2} \lambda_{j}^{2}+m_{2}^{2} N_{j}\right)}{\omega^{2} m_{3}^{2}}\right\} C_{j} \mathrm{e}^{i \omega\left(x-c t-\lambda_{j} z\right)} \\
\tau_{22}= & -\omega^{2} \sum_{j=1}^{3}\left\{\left[(\lambda+\alpha)\left(1+N_{j} \lambda_{j}\right)-\lambda_{j}\left(N_{j}-\lambda_{j}\right)-\frac{\gamma\left(1-i \omega c \tau_{1}\right)\left(m_{1}^{2}-\omega^{2} \lambda_{j}^{2}+m_{2}^{2} N_{j}\right)}{\omega^{2} m_{3}^{2}}\right]\right\} C_{j} \mathrm{e}^{i \omega\left(x-c t-\lambda_{j} z\right)} \\
\tau_{33}= & \omega^{2} \sum_{j=1}^{3}\left\{\left[\lambda_{j}^{2}\left(\lambda+2 \mu_{T}\right)-(\lambda+\alpha)-\frac{\gamma\left(1-i \omega c \tau_{1}\right)\left(m_{1}^{2}-\omega^{2} \lambda_{j}^{2}+m_{2}^{2} N_{j}\right)}{\omega^{2} m_{3}^{2}}\right]\right. \\
& \left.+\lambda_{j}\left[\left(\lambda+2 \mu_{T}\right)-(\lambda+\alpha)\right] N_{j}\right\} C_{j} \mathrm{e}^{i \omega\left(x-c t-\lambda_{j} z\right)} . \\
\tau_{12}= & i \omega \mu_{L} \sum_{j=1}^{3} G \mathrm{e}^{i \omega\left(x-c t-m_{6} z\right)} \\
\tau_{31}= & \mu_{L} \omega^{2} \sum_{j=1}^{3}\left\{2 \lambda_{j}+\left(\lambda_{j}^{2}-1\right) N_{j}\right\} C_{j} \mathrm{e}^{i \omega\left(x-c t-\lambda_{j} z\right)} \\
\tau_{32}= & -i \omega m_{6} \mu_{T} \sum_{j=1}^{3} G \mathrm{e}^{i \omega\left(x-c t-m_{6} z\right)}
\end{aligned}
$$

And for medium $M_{2}$

$$
u=i \omega \sum_{j=1}^{3}\left[1+N_{j}^{\prime} \lambda_{j}^{\prime}\right] C_{j}^{\prime} \mathrm{e}^{i \omega\left(x-c t-\lambda_{j}^{\prime} z\right)}
$$




$$
w=i \omega \sum_{j=1}^{3}\left[N_{j}^{\prime}-\lambda_{j}^{\prime}\right] C_{j}^{\prime} \mathrm{e}^{i \omega\left(x-c t-\lambda_{j}^{\prime} z\right)}
$$

And the stress components are

$$
\begin{aligned}
\tau_{11}= & \omega^{2} \sum_{j=1}^{3}\left\{\left(\lambda^{\prime}+\alpha^{\prime}\right)\left(\lambda_{j}^{\prime} N_{j}^{\prime}-\lambda_{j}^{\prime 2}\right)-\left(\lambda^{\prime}+2 \alpha^{\prime}+4 \mu_{L}^{\prime}-2 \mu_{T}^{\prime}+\beta^{\prime}\right)\left(1+N_{j}^{\prime} \lambda_{j}^{\prime}\right)\right. \\
& \left.-\frac{\gamma^{\prime}\left(1-i \omega c \tau_{1}\right)\left(m_{1}^{\prime 2}-\omega^{2} \lambda_{j}^{\prime 2}+m_{2}^{\prime 2} N_{j}^{\prime}\right)}{\omega^{2} m_{3}^{\prime 2}}\right\} C_{j} \mathrm{e}^{i \omega\left(x-c t-\lambda_{j}^{\prime} z\right)} \\
\tau_{22}= & -\omega^{2} \sum_{j=1}^{3}\left\{\left[\left(\lambda^{\prime}+\alpha^{\prime}\right)\left(1+N_{j}^{\prime} \lambda_{j}^{\prime}\right)-\lambda_{j}^{\prime}\left(N_{j}^{\prime}-\lambda_{j}^{\prime}\right)-\frac{\gamma^{\prime}\left(1-i \omega c \tau_{1}\right)\left(m_{1}^{\prime 2}-\omega^{2} \lambda_{j}^{\prime 2}+m_{2}^{\prime 2} N_{j}^{\prime}\right)}{\omega^{2} m_{3}^{\prime 2}}\right]\right\} C_{j} \mathrm{e}^{i \omega\left(x-c t-\lambda_{j}^{\prime} z\right)} \\
\tau_{33}= & \omega^{2} \sum_{j=1}^{3}\left\{\left[\lambda_{j}^{\prime 2}\left(\lambda^{\prime}+2 \mu_{T}^{\prime}\right)-\left(\lambda^{\prime}+\alpha^{\prime}\right)-\frac{\gamma^{\prime}\left(1-i \omega c \tau_{1}\right)\left(m_{1}^{\prime 2}-\omega^{2} \lambda_{j}^{\prime 2}+m_{2}^{\prime 2} N_{j}^{\prime}\right)}{\omega^{2} m_{3}^{\prime 2}}\right]\right. \\
& \left.+\lambda_{j}^{\prime}\left[\left(\lambda^{\prime}+2 \mu_{T}^{\prime}\right)-\left(\lambda^{\prime}+\alpha^{\prime}\right)\right] N_{j}^{\prime}\right\} C_{j}^{\prime} \mathrm{e}^{i \omega\left(x-c t-\lambda_{j}^{\prime} z\right)} \\
\tau_{12}= & i \omega \mu_{L}^{\prime} \sum_{j=1}^{3} G \mathrm{e}^{i \omega\left(x-c t-m_{6}^{\prime} z\right)} \\
\tau_{31}= & \mu_{L}^{\prime} \omega^{2} \sum_{j=1}^{3}\left\{2 \lambda_{j}^{\prime}+\left(\lambda_{j}^{\prime 2}-1\right) N_{j}^{\prime}\right\} C_{j}^{\prime} \mathrm{e}^{i \omega\left(x-c t-\lambda_{j}^{\prime} z\right)} \\
\tau_{32}= & i \omega m_{6}^{\prime} \mu_{T}^{\prime} \sum_{j=1}^{3} G \mathrm{e}^{i \omega\left(x-c t-m_{6}^{\prime} z\right)}
\end{aligned}
$$

\section{Boundary Conditions}

The boundary conditions for the titled problem are:

(1) The displacement components at the boundary surface between the media $M_{1}$ and $M_{2}$ must be continued at all times and positions.

i.e. $[u, v, w] M_{1}=[u, v, w] M_{2}$ at $z=0$ respectively.

(2) The stress components $\tau_{31}, \tau_{32}$ and $\tau_{33}$ must be continuous at the boundary $z=0$.

i.e. $\left[\tau_{31}, \tau_{32}, \tau_{33}\right] M_{1}=\left[\tau_{31}, \tau_{32}, \tau_{33}\right] M_{2}$, at $z=0$ respectively.

(3) The thermal boundary conditions must be continuous at the boundary $z=0$.

i.e. $\left\{\begin{array}{l}\left(\frac{\partial T}{\partial z}+h T\right)_{M_{1}}=\left(\frac{\partial T}{\partial z}+h T\right)_{M_{2}} \text {, at } z=0 . \\ T_{M_{1}}=T_{M_{2}}\end{array}\right.$

Applying the boundary conditions (1)-(3), we have

$$
\begin{aligned}
& \left(1+N_{j} \lambda_{j}\right) C_{j}=\left(1+N_{j}^{\prime} \lambda_{j}^{\prime}\right) C_{j}^{\prime}, \\
& G=G^{\prime}, \\
& \left(\lambda_{j}-N_{j}\right) C_{j}=\left(\lambda_{j}^{\prime}-N_{j}^{\prime}\right) C_{j}^{\prime}, \\
& \mu_{L}\left\{2 \lambda_{j}+\left(\lambda_{j}^{2}-1\right) N_{j}\right\} C_{j}=\mu_{L}^{\prime}\left\{2 \lambda_{j}^{\prime}+\left(\lambda_{j}^{\prime 2}-1\right) N_{j}^{\prime}\right\} C_{j}^{\prime} \\
& \mu_{T} m_{6} G=\mu_{T}^{\prime} m_{6}^{\prime} G^{\prime}
\end{aligned}
$$




$$
\begin{aligned}
& {\left[\lambda_{j}\left(\lambda+2 \mu_{T}\right)\left[\lambda_{j}+N_{j}\right]-(\lambda+\alpha)\left[1+\lambda_{j} N_{j}\right]-\frac{\gamma\left(1-i \omega c \tau_{1}\right)\left(m_{1}^{2}-\omega^{2} \lambda_{j}^{2}+m_{2}^{2} N_{j}\right)}{\omega^{2} m_{3}^{2}}\right] C_{j}} \\
& =\left[\lambda_{j}^{\prime}\left(\lambda^{\prime}+2 \mu_{T}^{\prime}\right)\left[\lambda_{j}^{\prime}+N_{j}^{\prime}\right]-\left(\lambda^{\prime}+\alpha^{\prime}\right)\left[1+\lambda_{j}^{\prime} N_{j}^{\prime}\right]-\frac{\gamma^{\prime}\left(1-i \omega c \tau_{1}\right)\left(m_{1}^{\prime 2}-\omega^{2} \lambda_{j}^{\prime 2}+m_{2}^{\prime 2} N_{j}^{\prime}\right)}{\omega^{2} m_{3}^{\prime 2}}\right] C_{j}^{\prime}, \\
& \frac{1}{m_{3}^{2}}\left(m_{1}^{2}-\omega^{2} \lambda_{j}^{2}+m_{2}^{2} N_{j}\right)\left[h-i \omega \lambda_{j}\right] C_{j}=\frac{1}{m_{3}^{\prime 2}}\left(m_{1}^{\prime 2}-\omega^{2} \lambda_{j}^{\prime 2}+m_{2}^{\prime 2} N_{j}^{\prime}\right)\left[h-i \omega \lambda_{j}^{\prime}\right] C_{j}^{\prime}, \\
& \frac{1}{m_{3}^{2}}\left(m_{1}^{2}-\omega^{2} \lambda_{j}^{2}+m_{2}^{2} N_{j}\right) C_{j}=\frac{1}{m_{3}^{\prime 2}}\left(m_{1}^{\prime 2}-\omega^{2} \lambda_{j}^{\prime 2}+m_{2}^{\prime 2} N_{j}^{\prime}\right) C_{j}^{\prime} .
\end{aligned}
$$

From Equations (53) and (56), we have $G=G^{\prime}=0$. Thus there is no propagation of displacement $v$. The constants $C_{j}$ and $C_{j}^{\prime}$ we can determinate where $j=1,2,3$.

The discussion in two fibre-reinforced anisotropic solid thermoelastic under effect thermal relaxation time and gravity is clear up from Figures 1-4 and dissection some special cases is clear up from Figures 5-12.

\section{Particular Cases}

\subsection{Isotropic Generalized Thermoelastic Medium with Gravity}

In this case, substituting $\mu_{L}=\mu_{T}=\mu, \gamma=\alpha_{t}(3 \lambda+2 \mu)$ and $\beta=0$ in Equations (47)-(51), we obtain the corresponding expressions of displacement, temperature and stress in isotropic generalized thermoelastic medium with gravity.

\subsection{Fibre-Reinforced Anisotropic Generalized Thermoelastic Medium with Neglected the Gravity}

In this case, letting $g=0$, in Equations (47)-(51), we obtain the corresponding expressions of displacement, temperature and stress in anisotropic generalized thermoelastic medium with neglected the gravity.

\subsection{Isotropic Generalized Thermoelastic Medium with Neglected the Gravity}

In this case, substituting $\mu_{L}=\mu_{T}=\mu, \gamma=\alpha_{t}(3 \lambda+2 \mu), \beta=0$ and $g=0$ in Equations (47)-(51), we obtain the corresponding expressions of displacement, temperature and stress in isotropic generalized thermoelastic medium with neglected the gravity.

\section{Numerical Results and Discussion}

The following values of elastic constants are considered Singh [25] and Chattopadhyay [26], for mediums $M_{1}$ and $M_{2}$ respectively.

$$
\begin{gathered}
\rho=2660 \mathrm{~kg} / \mathrm{m}^{3}, \lambda=5.65 \times 10^{10} \mathrm{~N} \cdot \mathrm{m}^{-2}, \mu_{T}=2.46 \times 10^{9} \mathrm{~N} \cdot \mathrm{m}^{-2}, \\
\mu_{L}=5.66 \times 10^{9} \mathrm{~N} \cdot \mathrm{m}^{-2}, \alpha=-1.28 \times 10^{9} \mathrm{~N} \cdot \mathrm{m}^{-2}, \beta=220.90 \times 10^{9} \mathrm{~N} \cdot \mathrm{m}^{-2}, \\
\rho=7800 \mathrm{~kg} / \mathrm{m}^{3}, \lambda=5.65 \times 10^{9} \mathrm{~N} \cdot \mathrm{m}^{-2}, \mu_{T}=2.46 \times 10^{10} \mathrm{~N} \cdot \mathrm{m}^{-2}, \\
\mu_{L}=5.66 \times 10^{10} \mathrm{~N} \cdot \mathrm{m}^{-2}, \alpha=-1.28 \times 10^{10} \mathrm{~N} \cdot \mathrm{m}^{-2}, \beta=220.90 \times 10^{10} \mathrm{~N} \cdot \mathrm{m}^{-2}, \\
c_{v}=0.787 \times 10^{3} \mathrm{~J} / \mathrm{kg} \cdot \mathrm{K}, \mathrm{K}=0.0921 \times 10^{3} \mathrm{~J} \cdot \mathrm{m}^{-1} \cdot \mathrm{deg}^{-1} \cdot \mathrm{s}^{-1}, T_{0}=293 \mathrm{~K}, c=1.2 \times 10^{4} \mathrm{~m}^{2} / \mathrm{s}^{2} \cdot \mathrm{K} .
\end{gathered}
$$

The numerical technique outlined above was used to obtain the displacement, temperature and thermal stresses under effect of thermal relaxation time and gravity. These distributions are shown in Figures 1-12 respectively.

-In two fibre-reinforced anisotropic solid thermoelastic under effect thermal relaxation time and gravity

From Figure 1: For medium $M_{1}$ it is seen that the displacements, normal stress $\tau_{33}$ and shear stress $\tau_{31}$ increase but the temperature and normal stresses $\tau_{11}, \tau_{22}$ decrease with an increasing the gravity $g$. 


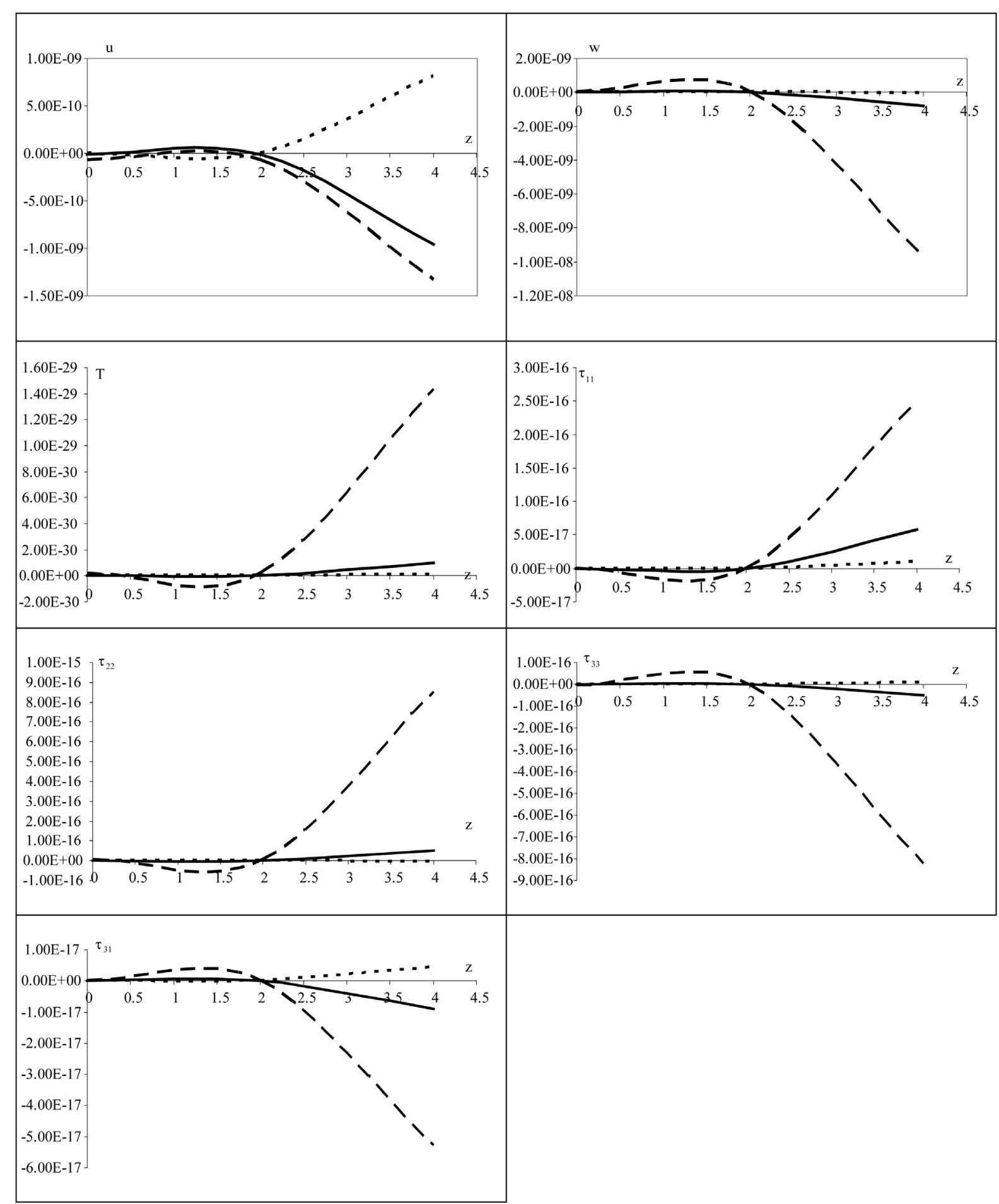

Figure 1. Effects of $g$ on displacements $u, w$, temperature $T$ and thermal stresses with horizontal distance $\mathrm{z}$ for medium $M_{1}$. --- --- --- $g=0.1$, $g=0.3, \ldots \ldots \ldots . . . g=0.5$.

From Figure 2: For medium $M_{2}$ it is seen that the displacements, normal stresses $\tau_{11}$, $\tau_{33}$ and shear stress $\tau_{31}$ increase but the normal stresses $\tau_{22}$ decrease with an increasing the gravity $g$. Also, a slight increase in the temperature with an increasing the gravity $g$.

From Figure 3: For medium $M_{1}$ it is seen that the displacements, temperature, normal stresses $\tau_{22}$, $\tau_{33}$ and 


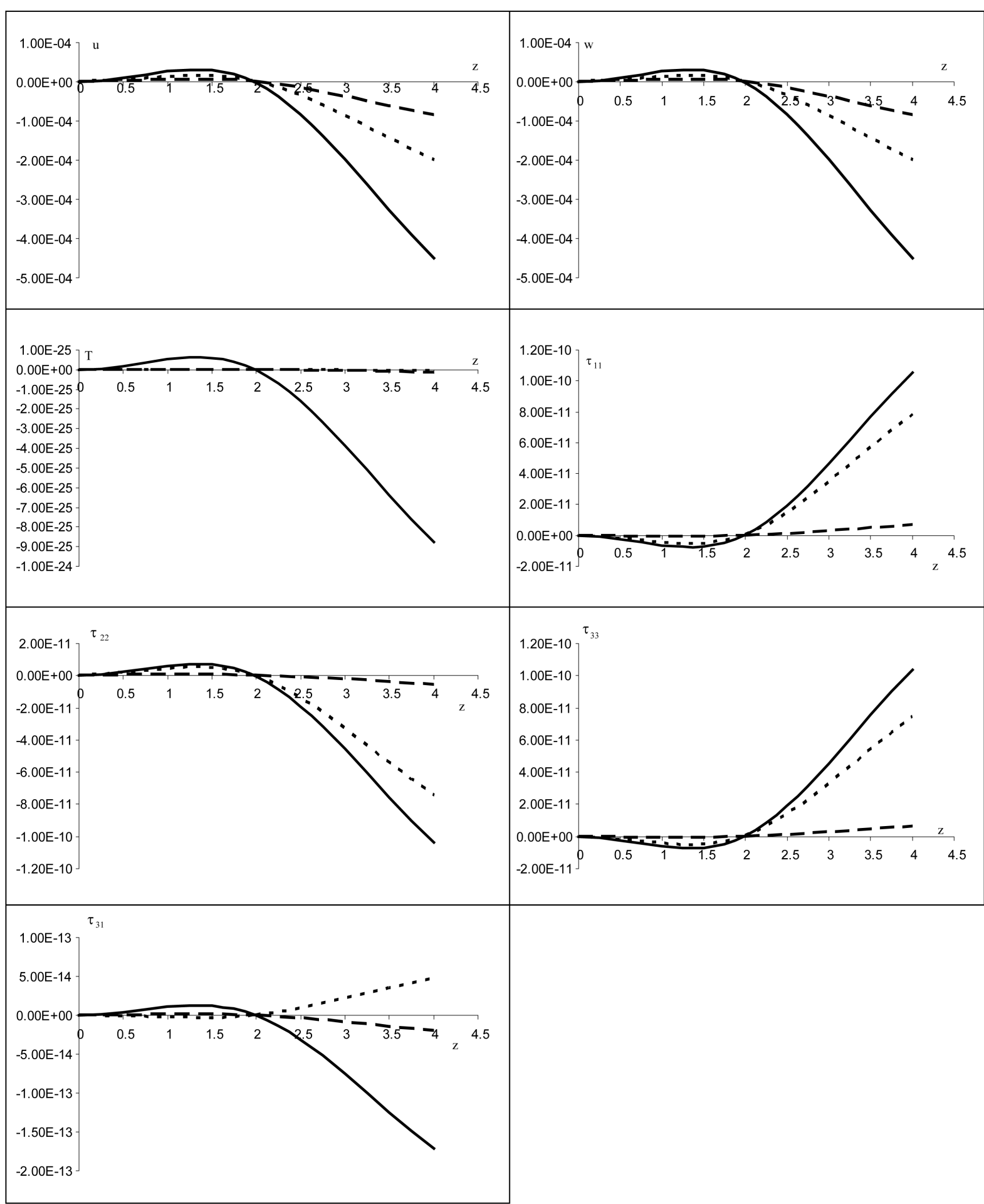

Figure 2. Effects of $g$ on displacements $u, w$, temperature $T$ and thermal stresses with horizontal distance $z$ for medium $M_{2}$. --- --- ---g $g=0.1, \ldots g=0.3, \ldots \ldots \ldots \ldots . . . \quad g=0.5$.

shear stress $\tau_{31}$ increase but the normal stresses $\tau_{11}$ decrease with an increasing the thermal relaxation time $\tau_{2}$.

From Figure 4: For medium $M_{2}$ it is seen that the displacement $u$, temperature, normal stresses $\tau_{11}, \tau_{33}$ and shear stress $\tau_{31}$ increase but the displacement $w$ and normal stresses $\tau_{22}$ decrease with an increasing the thermal relaxation time $\tau_{2}$. 


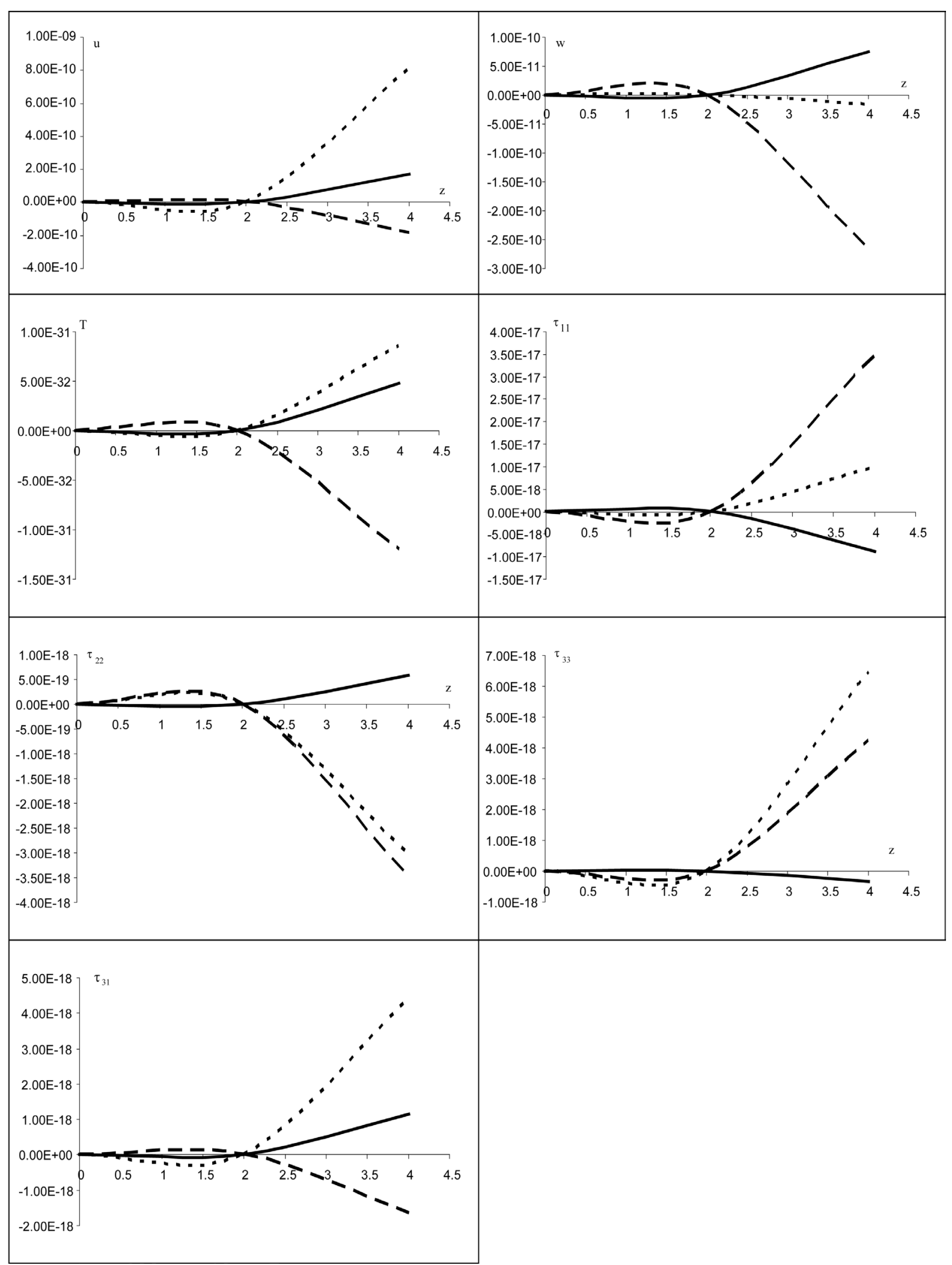

Figure 3. Effects of $\tau_{2}$ on displacements $u, w$, temperature $T$ and thermal stresses with horizontal distance $z$ for medium $M_{1}$.-- --- ---- $\tau_{2}=0.2$, $\tau_{2}=0.3, \ldots \ldots \ldots \ldots, \tau_{2}=0.4$ 


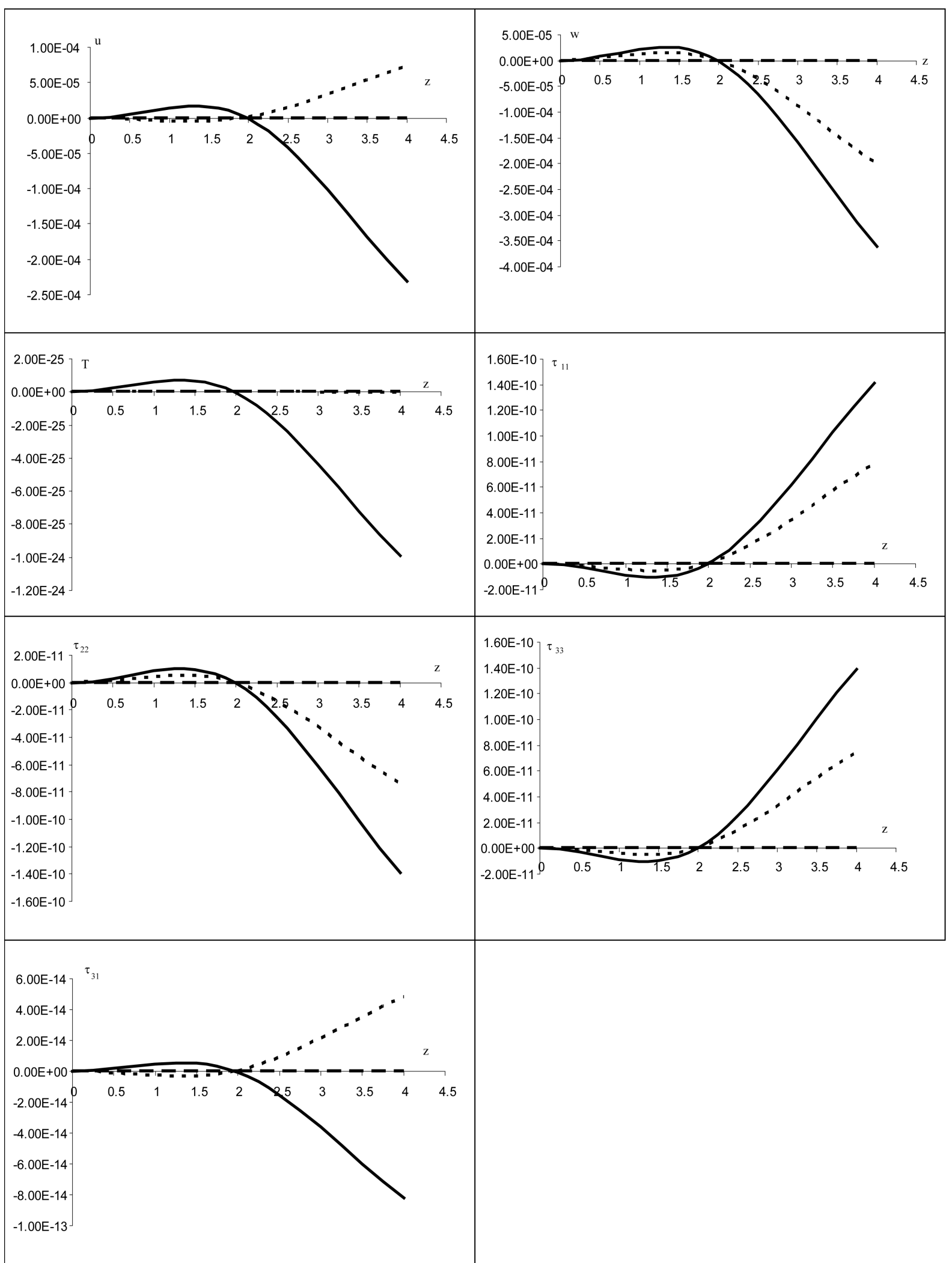

Figure 4. Effects of $\tau_{2}$ on displacements $u, w$, temperature $T$ and thermal stresses with horizontal distance $z$ for medium $M_{2 .---}---\quad----\tau_{2}=0.2$, $\tau_{2}=0.3$ $\tau_{2}=0.4$. 


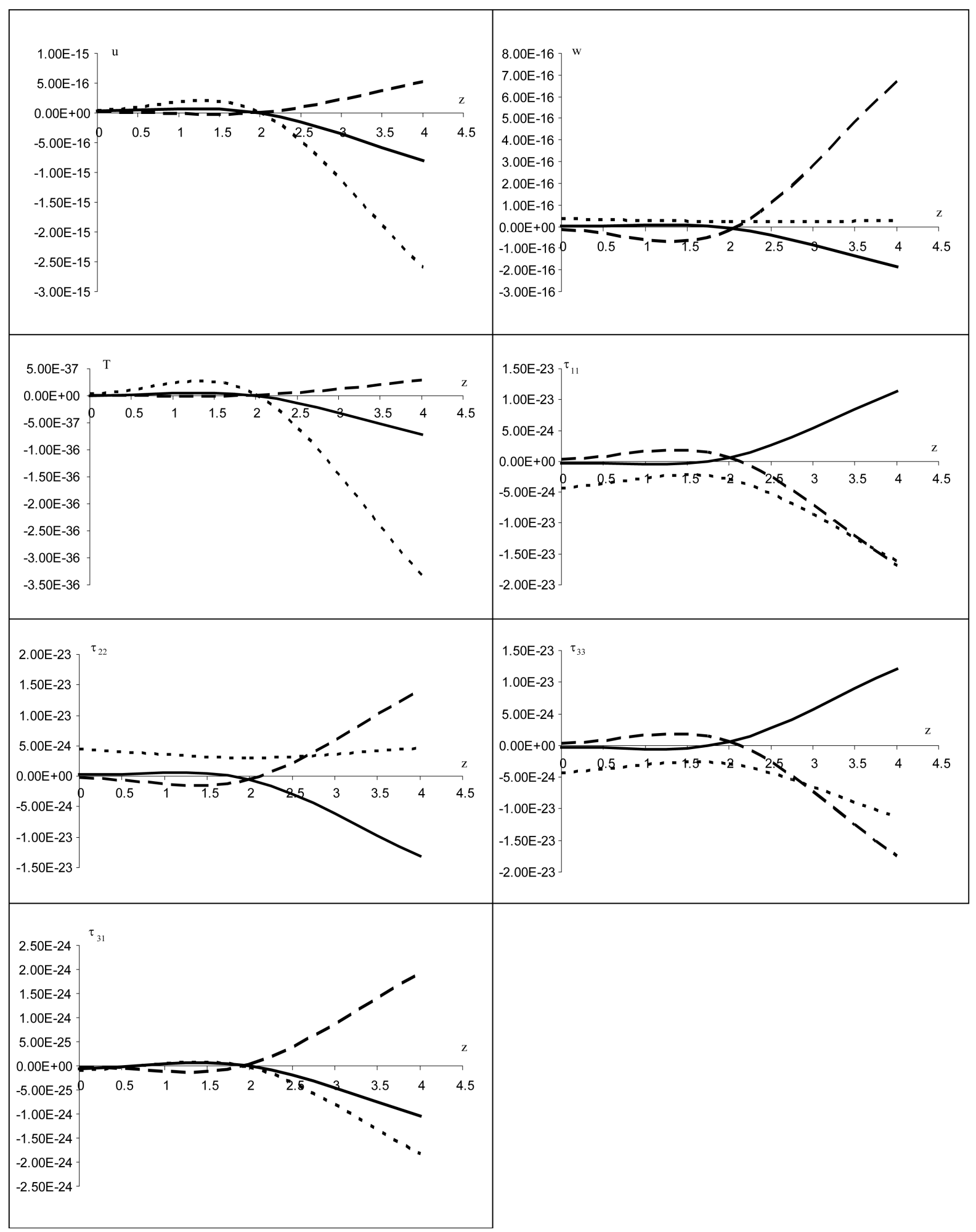

Figure 5. Effects of $g$ on displacements $u, w$, temperature $T$ and thermal stresses with horizontal distance $\mathrm{z}$ for medium $M_{1}$. $-------g=0.1$, $g=0.3, \ldots \ldots \ldots . . . . g=0.5$.

-In two fibre-reinforced isotropic solid thermoelastic under effect thermal relaxation time and gravity From Figure 5: For medium $M_{1}$ it is seen that the displacements, temperature, normal stresses $\tau_{11}$, $\tau_{33}$ and 


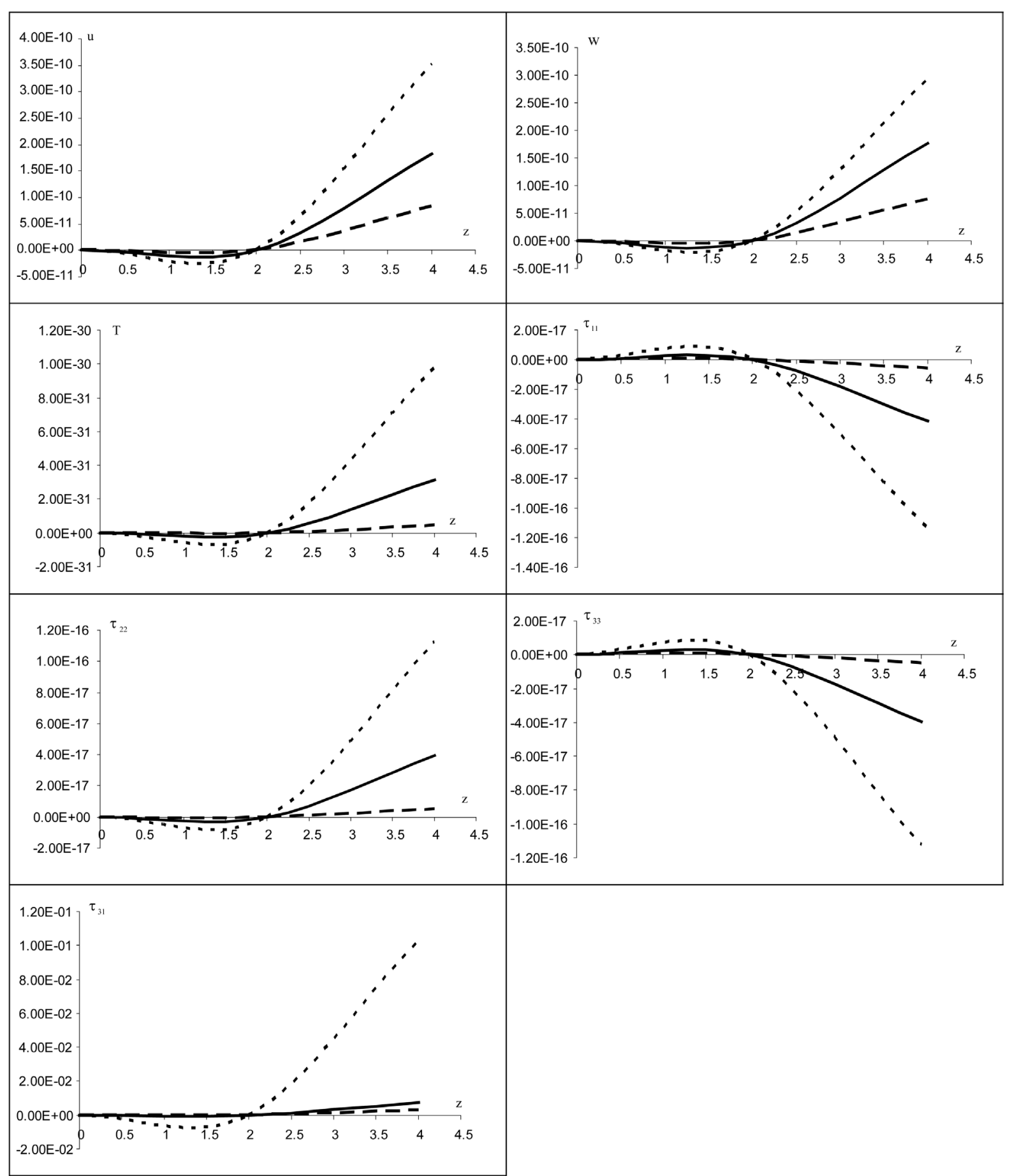

Figure 6. Effects of $g$ on displacements $u, w$, temperature $T$ and thermal stresses with horizontal distance $z$ for medium $M_{2}$. --- ---- $g=0.1, \ldots$

shear stress $\tau_{31}$ decrease but the normal stress $\tau_{22}$ increase with an increasing the gravity $g$.

From Figure 6: For medium $M_{2}$ it is seen that the displacements, temperature, normal stress $\tau_{22}$ and shear stress $\tau_{31}$ increase but the normal stresses $\tau_{11}, \tau_{33}$ decrease with an increasing the gravity $g$.

From Figure 7: For medium $M_{1}$ it is seen that the displacements, temperature and normal stress $\tau_{11}$ decrease but the normal stresses $\tau_{22}, \tau_{33}$ and shear stress $\tau_{31}$ increase with an increasing the thermal relaxation time $\tau_{2}$.

From Figure 8: For medium $M_{2}$ it is seen that the displacement $u$, temperature and normal stress $\tau_{11}$, $\tau_{33}$ 


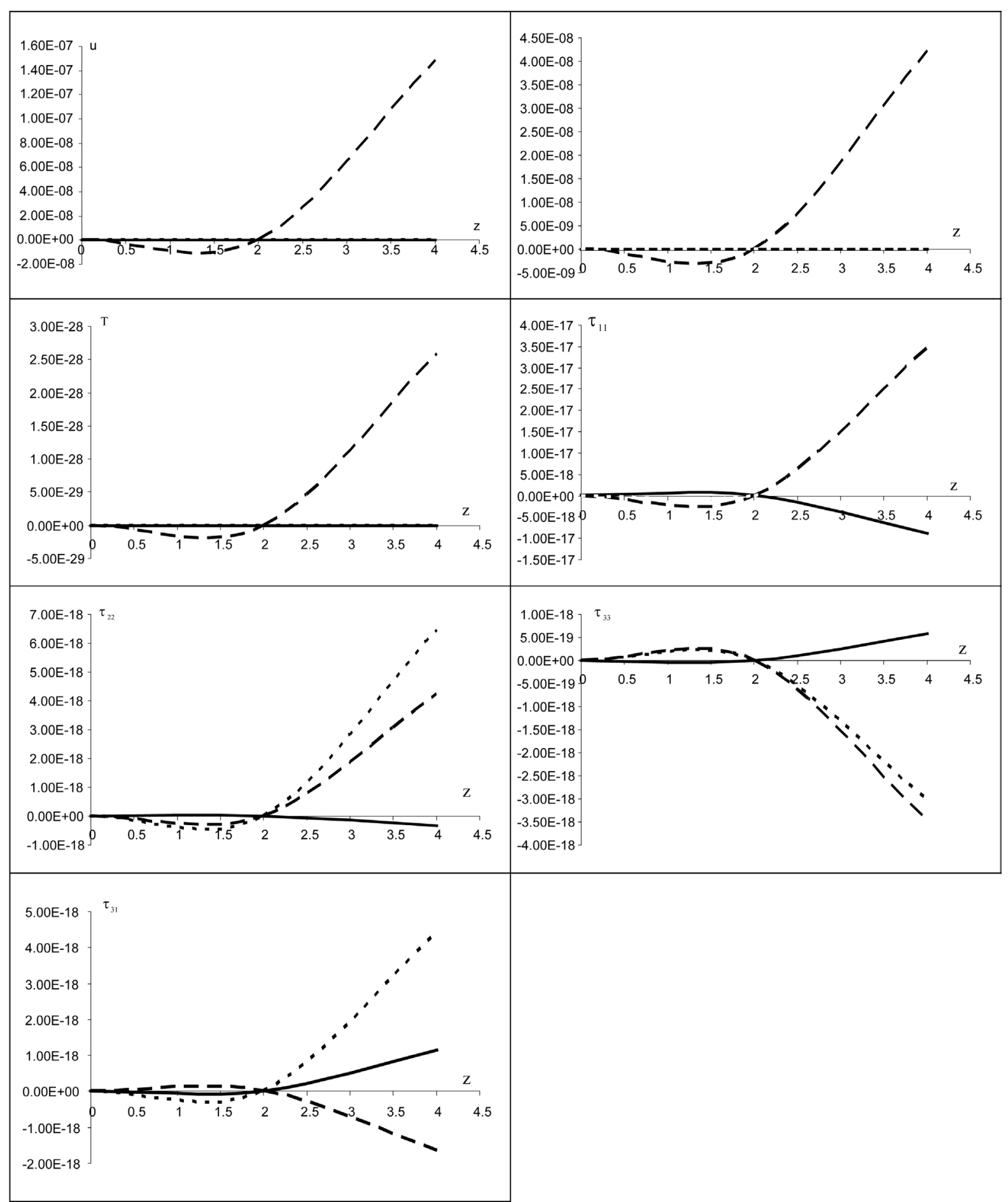

Figure 7. Effects of $\tau_{2}$ on displacements $u, w$, temperature $T$ and thermal stresses with horizontal distance $z$ for medium $M_{1}$.--- --- $\quad----\tau_{2}=0.2$, $\tau_{2}=0.3$ $\tau_{2}=0.4$.

and shear stress $\tau_{31}$ increase but the displacement $w$, normal stress $\tau_{22}$ decrease with an increasing the thermal relaxation time $\tau_{2}$.

-In two fibre-reinforced anisotropic generalized thermoelastic medium with neglected the gravity

From Figure 9: For medium $M_{1}$ it is seen that the displacement $w$, temperature and normal stress $\tau_{33}$ and 


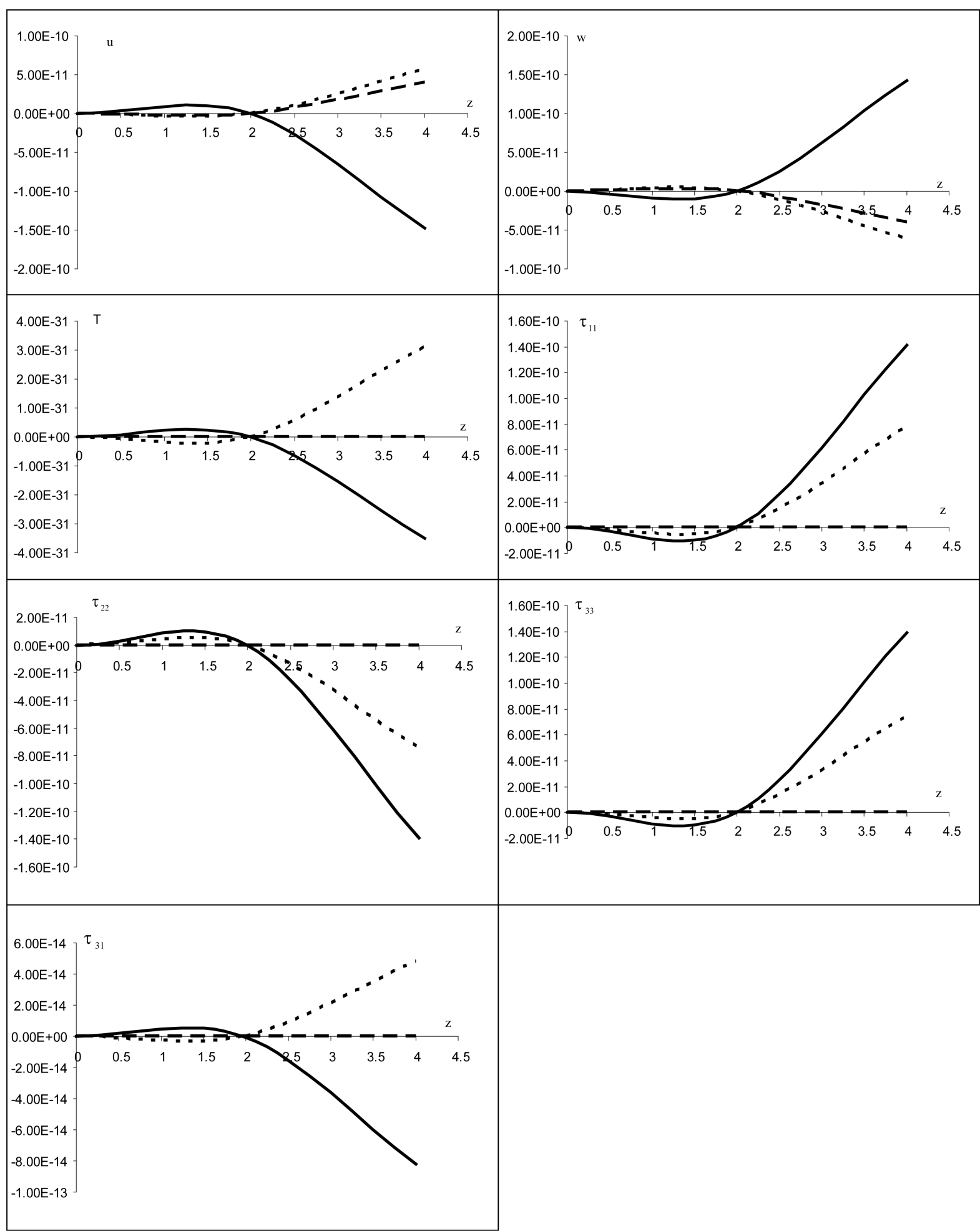

Figure 8. Effects of $\tau_{2}$ on displacements $u, w$, temperature $T$ and thermal stresses with horizontal distance $z$ for medium $M_{2}$--- --- $----\tau_{2}=0.2$, $\tau_{2}=0.4$.

shear stress $\tau_{31}$ increase but the displacement $u$, normal stresses $\tau_{11}$, $\tau_{22}$ decrease with an increasing the thermal relaxation time $\tau_{2}$. 
F. S. Bayones, N. S. Hussien

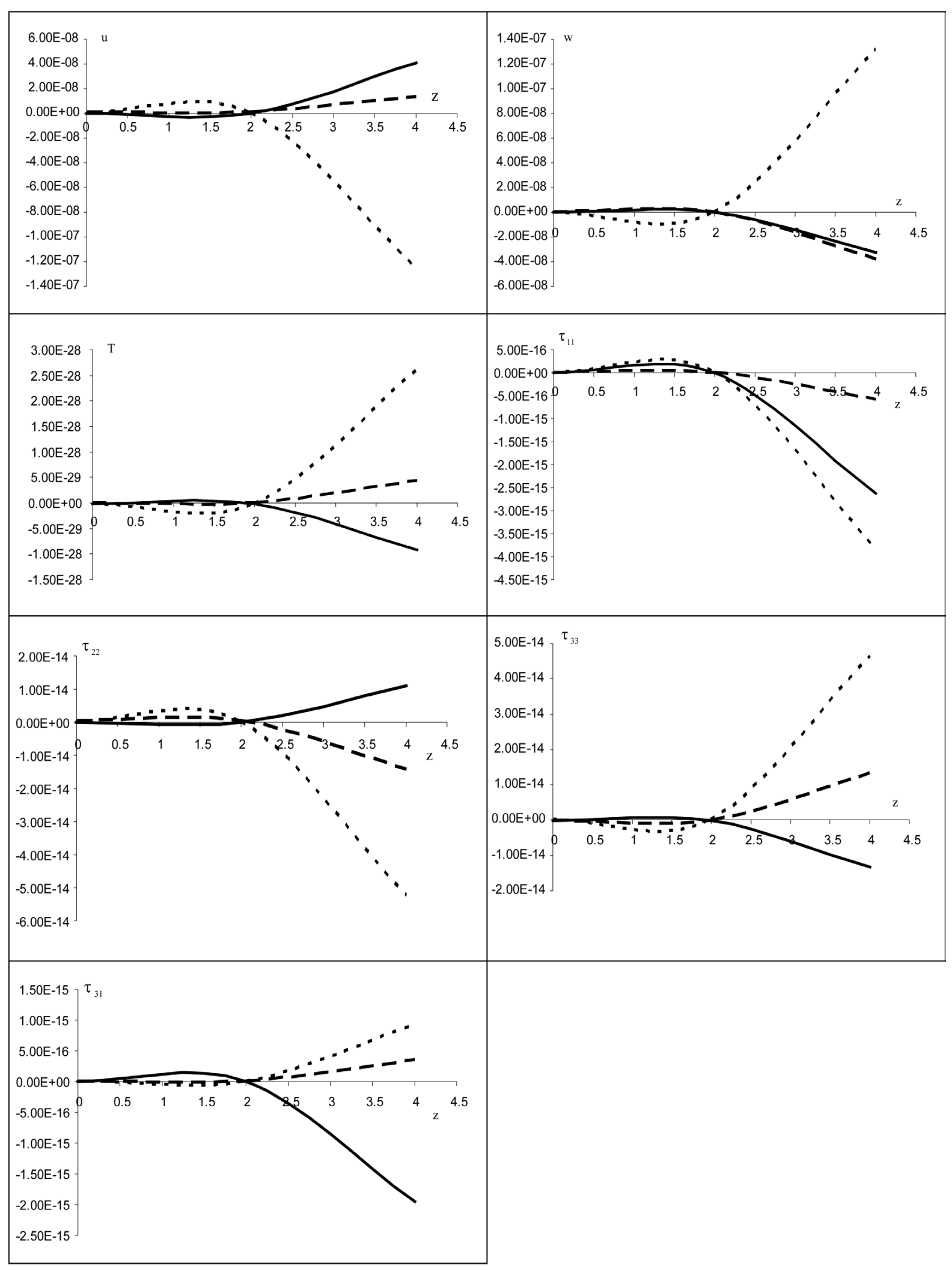

Figure 9. Effects of $\tau_{2}$ on displacements $u, w$, temperature $T$ and thermal stresses with horizontal distance $z$ for medium $M_{1}$.--- --- --- $\tau_{2}=0.2$, $\tau_{2}=0.3$, $\tau_{2}=0.4$. 


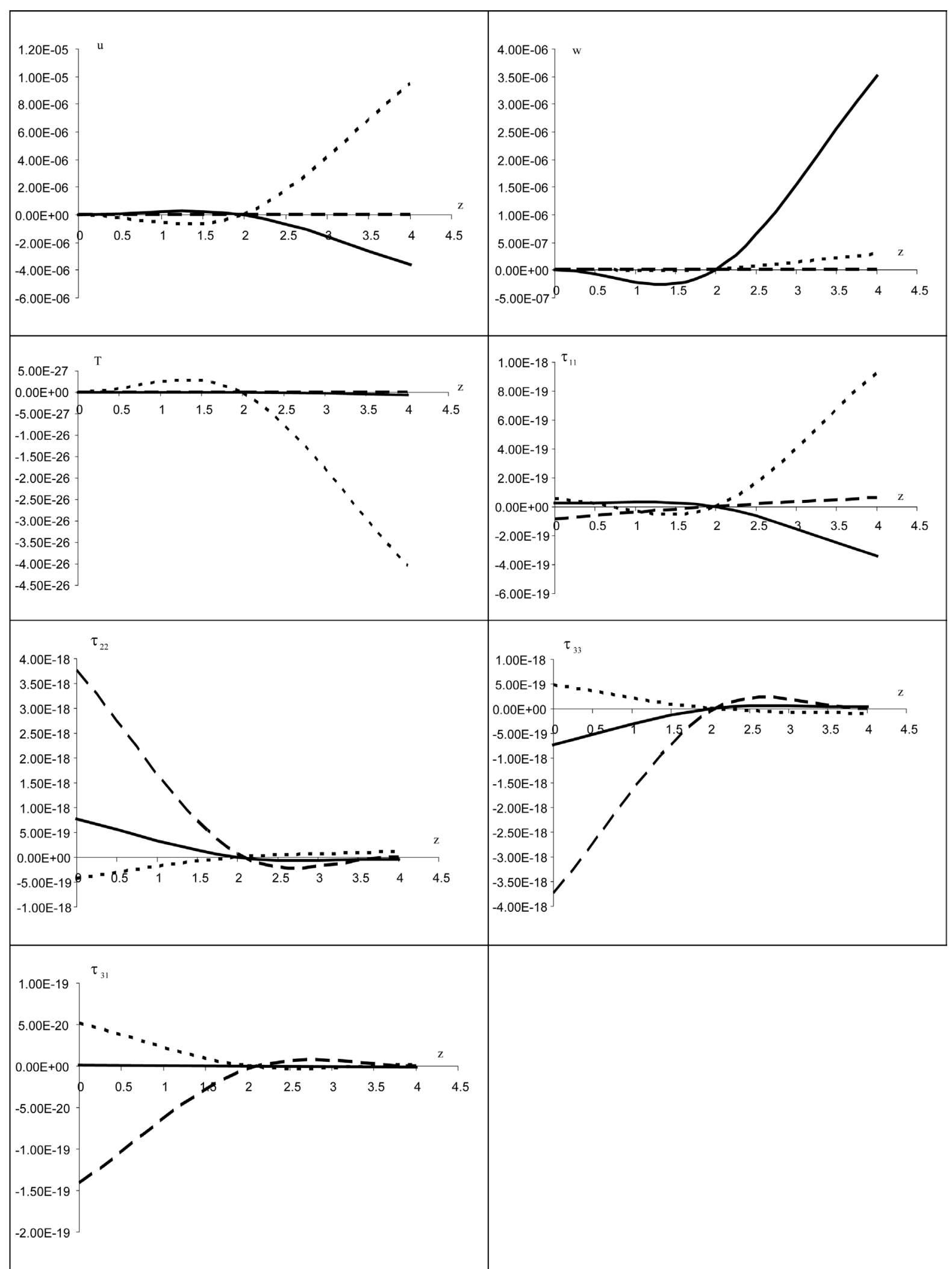

Figure 10. Effects of $\tau_{2}$ on displacements $u, w$, temperature $T$ and thermal stresses with horizontal distance $\mathrm{z}$ for medium $M_{2}$. --- $\quad---\quad---\tau_{2}=0.2$, $\tau_{2}=0.3$, $\tau_{2}=0.4$. 


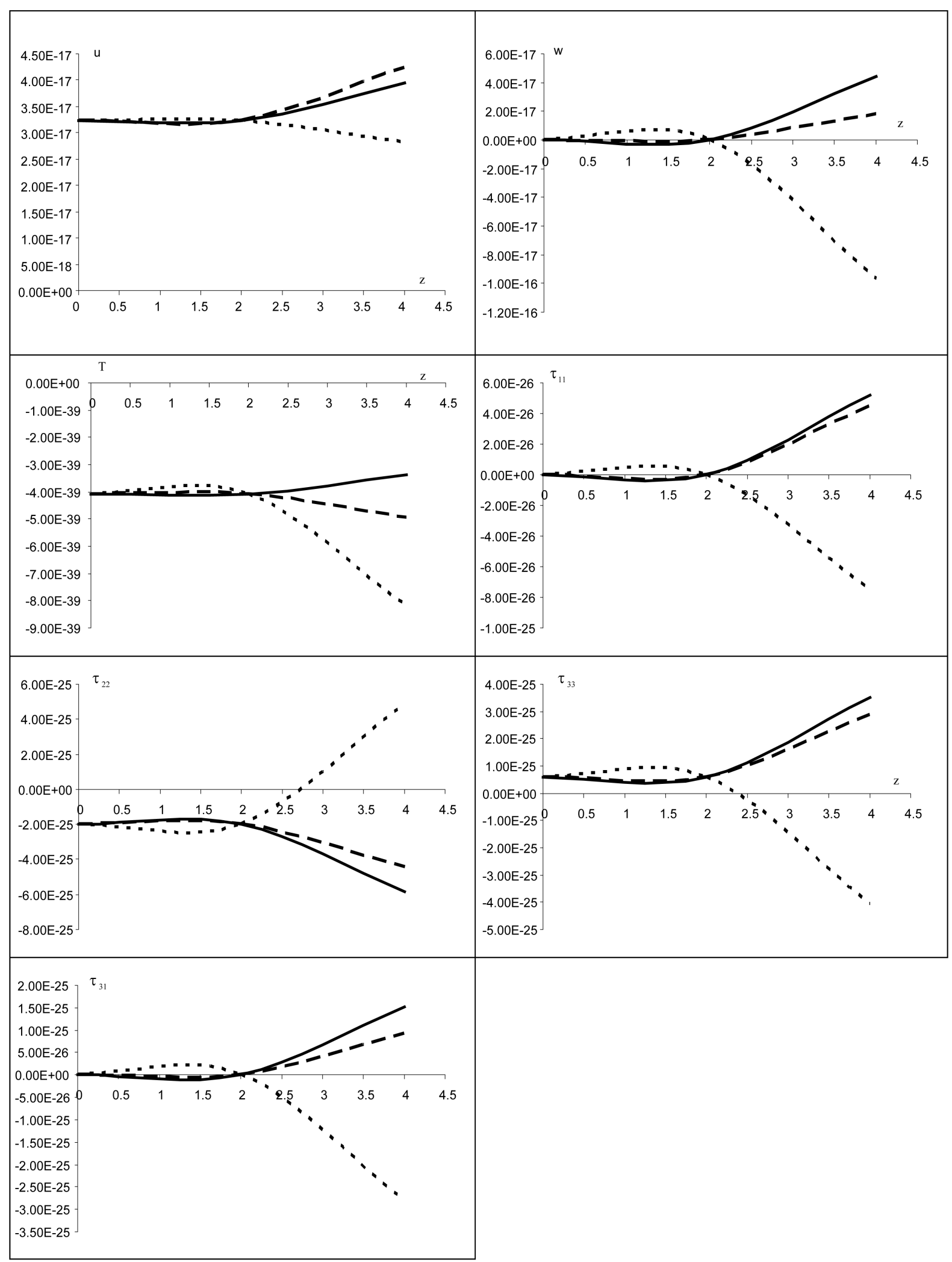

Figure 11. Effects of $\tau_{2}$ on displacements $u, w$, temperature $T$ and thermal stresses with horizontal distance $z$ for medium $M_{1}$ - --- --- $\quad----\tau_{2}=0.2$, $\tau_{2}=0.3, \ldots \ldots \ldots \ldots, \tau_{2}=0.4$. 


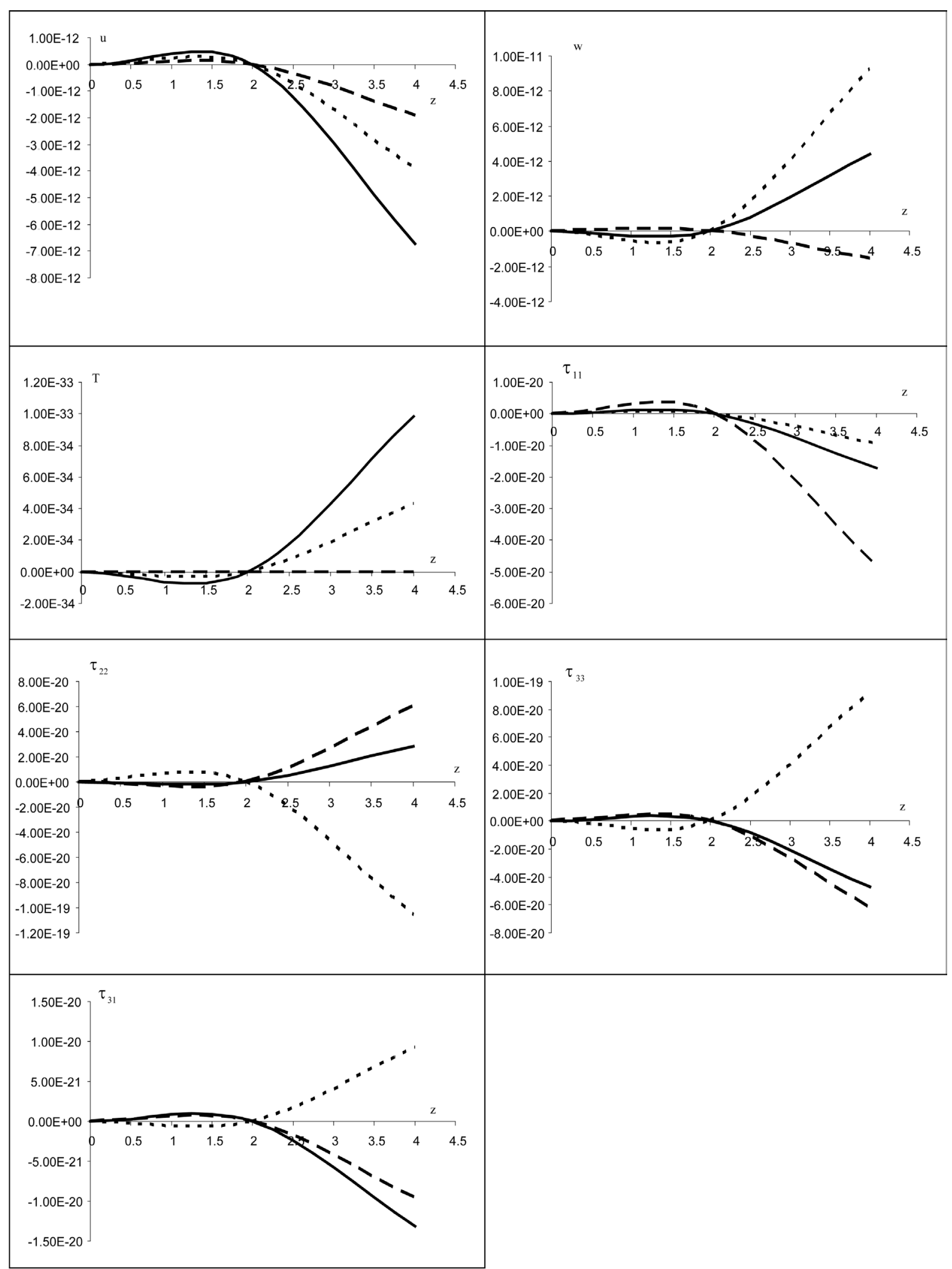

Figure 12. Effects of $\tau_{2}$ on displacements $u, w$, temperature $T$ and thermal stresses with horizontal distance $z$ for medium $M_{2}$. - -- $\quad$--- $\quad$--- $\tau_{2}=0.2, \ldots \tau_{2}=0.3, \ldots \ldots \ldots \ldots . . \quad \tau_{2}=0.4$. 
From Figure 10: For medium $M_{2}$ it is seen that the displacements and normal stresses $\tau_{11}, \tau_{22}$ increase but the temperature, normal stress $\tau_{33}$ and shear stress $\tau_{31}$ decrease with an increasing the thermal relaxation time $\tau_{2}$.

-In two fibre-reinforced isotropic generalized thermoelastic medium with neglected the gravity

From Figure 11: For medium $M_{1}$ it is seen that the displacements, temperature and normal stresses $\tau_{11}, \tau_{33}$ and shear stress $\tau_{31}$ decrease but the normal stress, $\tau_{22}$ increase with an increasing the thermal relaxation time $\tau_{2}$.

From Figure 12: For medium $M_{2}$ it is seen that the displacement $w$, temperature, normal stresses $\tau_{11}, \tau_{33}$ and shear stress $\tau_{31}$ increase but the displacement $u$ and normal stress $\tau_{22}$ decrease with an increasing the thermal relaxation time $\tau_{2}$.

\section{Conclusions}

Due to the complicated nature of the governing equations of the generalized thermoelasticity fiber-reinforced theory, the work done in this field is unfortunately limited in number. The method used in this study provides a quite successful in dealing with such problems. This method gives exact solutions in the generalized thermoelastic medium without any assumed restrictions on the actual physical quantities that appear in the governing equations of the problem considered. Important phenomena are observed in all these computations:

- It was found that for large values of relaxation time they give close results. The solutions obtained in the context of generalized thermoelasticity theory, however, exhibit the behavior of the components of displacement, stresses and temperature.

- By comparing Figures 1-12, it was found that the surface wave velocity has the same behavior in both media. But with the passage of relaxation times and gravity, numerical values of the components of displacement, stresses and temperature in the thermoelastic medium are large in comparison due to the influences of gravity and relaxation times.

- Special cases are considered as isotropic generalized thermoelastic medium with gravity and fibre-reinforced anisotropic generalized thermoelastic medium with neglected the gravity in anisotropic generalized thermoelastic medium, as well in the isotropic case.

- The results presented in this paper should prove useful for researchers in material science, designers of new materials.

- Study of the phenomenon of rotation and gravity is also used to improve the conditions of oil extractions.

\section{References}

[1] Biot, M.A. (1956) Journal of Applied Physics, 27, 240-253. http://dx.doi.org/10.1063/1.1722351

[2] Lord, H. and Shulman, Y. (1967) Journal of the Mechanics and Physics of Solids, 15, 299-309. http://dx.doi.org/10.1016/0022-5096(67)90024-5

[3] Green, A.E. and Lindsay, K.A. (1972) Journal of Elasticity, 2, 1-7. http://dx.doi.org/10.1007/BF00045689

[4] Tanaka, M., Matsumoto, M. and Moradi, M. (1995) Engineering Analysis with Boundary Element, 16, $297-303$. http://dx.doi.org/10.1016/0955-7997(95)00074-7

[5] Green, A.E. and Laws, N. (1972) Archives of Rational Mechanics and Analysis, 45, 45-47. http://dx.doi.org/10.1007/BF00253395

[6] Abd-Alla, A.M., Abd-Alla, A.N. and Zeidan, N.A. (2001) Applied Mathematics and Computation, 121, 93-122. http://dx.doi.org/10.1016/S0096-3003(99)00265-9

[7] El-Naggar, A.M. and Abd-Alla, A.M. (1987) Earths, Moon Planets, 37, 213-223. http://dx.doi.org/10.1007/BF00116637

[8] Abd-Alla, A.M., Abo-Bahab, S.M. and Bayones, F.S. (2011) International Review of Physics, 5, 171-181.

[9] Ezzat, M.A. and Youssef, H.M. (2005) International Journal of Solids and Structures, 42, 6319-6334. http://dx.doi.org/10.1016/j.ijsolstr.2005.03.065

[10] Abd-Alla, A.M. and Bayones, F.S. (2011) Applied Mathematical Sciences, 5, 2049-2076.

[11] Dai, H.L. and Wang, X. (2006) International Journal of Engineering Science, 44, 365-378. http://dx.doi.org/10.1016/j.ijengsci.2005.12.008

[12] Abd-Alla, A.M., Abd-Alla, A.N. and Zeidan, N.A. (2000) Journal of Thermal Stresses, 23, 413-428. 
http://dx.doi.org/10.1080/014957300403914

[13] Craig, M.S. and Hart, V.G. (1979) Quarterly Journal of Mechanics and Applied Mathematics, 4, 473-498. http://dx.doi.org/10.1093/qimam/32.4.473

[14] Sengupta, P.R. and Nath, S. (2001) Sadhana, 26, 363-370. http://dx.doi.org/10.1007/BF02703405

[15] Singh, B. and Singh, S.J. (2004) Sadhana, 29, 249-257. http://dx.doi.org/10.1007/BF02703774

[16] Singh, B. (2007) Archives of Applied Mechanics, 77, 253-258. http://dx.doi.org/10.1007/s00419-006-0094-9

[17] Singh, B. (2007) International Journal of Mechanics and Solids, 2, 39-49.

[18] Kumar, R. and Gupta, R. (2009) Multidiscipline Modeling in Materials and Structures, 5, 283-288. http://dx.doi.org/10.1163/157361109789017014

[19] Abd-Alla, A.M., Abo-Dahab, S.M. and Hammad, H.A.H. (2011) Applied Mathematical Modelling, 35, $2981-3000$. http://dx.doi.org/10.1016/j.apm.2010.11.067

[20] Abd-Alla, A.M., Yahya, G.A. and Farhan, A.M. (2012) Journal of Mechanical Science and Technology, 26, $1829-1839$. http://dx.doi.org/10.1007/s12206-012-0424-5

[21] Abd-Alla, A.M. and Mahmoud, S.R. (2010) Meccanica, 45, 451-462. http://dx.doi.org/10.1007/s11012-009-9261-8

[22] Abd-Alla, A.M. and Ahmed, S.M. (1996) Earth, Moon, and Planets, 75, 185-197. http://dx.doi.org/10.1007/BF02592996

[23] Abd-Alla, A.M., Hammad, H.A.H. and Abo-Dahab, S.M. (2004) Applied Mathematics and Computation, 154, $583-597$. http://dx.doi.org/10.1016/S0096-3003(03)00767-7

[24] Elnaggar A.M. and Abd-Alla, A.M. (1989) Earth, Moon, and Planets, 45, 175-185. http://dx.doi.org/10.1007/BF00055784

[25] Abd-Alla, A.M. and Ahmed, S.M. (1996) Earth, Moon, and Planets, 75, 185-197. http://dx.doi.org/10.1007/BF02592996

[26] Abd-Alla, A.M. and Abo-Dahab, S.M. (2012) Journal of Thermal Stresses, 35, 892-912. http://dx.doi.org/10.1080/01495739.2012.720209 